\title{
Nonstationary pearl pulsations as a signature of magnetospheric disturbances
}

\author{
F. Z. Feygin ${ }^{1}$, N. G. Kleimenova ${ }^{1}$, O. A. Pokhotelov ${ }^{1}$, M. Parrot ${ }^{2}$, \\ K. Prikner ${ }^{3}$, K. Mursula ${ }^{4}$, J. Kangas ${ }^{5}$, T. Pikkarainen ${ }^{4}$ \\ ${ }^{1}$ Institute of Physics of the Earth, Moscow, 123810, Russia \\ ${ }^{2}$ Laboratoire de Physique et Chimie de l'Environnement, Centre National de la Recherche Scientifique, Orléans, France \\ ${ }^{3}$ Geophysical Institute, Czech Academy of Sciences, Prague, Czech Republic \\ ${ }^{4}$ Department of Physical Sciences, P.O. Box 3000, FIN-90014 University of Oulu, Finland \\ ${ }^{5}$ Sodankylä Geophysical Observatory, FIN-99600 Sodankylä, Finland
}

Received: 3 May 1999 / Revised: 24 January 2000 / Accepted: 9 February 2000

\begin{abstract}
We analyse long-lasting (several hours) Pc1 pearl pulsations with decreasing, increasing or constant central frequencies. We show that nonstationary pearl events (those with either decreasing or increasing central frequency) are observed simultaneously with increasing auroral magnetic activity at the nightside magnetosphere while the stationary events (constant central frequency) correspond to quiet magnetic conditions. Events with decreasing central frequency are observed mostly in the late morning and daytime whereas events with increasing central frequency appear either early in the morning or in the afternoon. We explain the diurnal distribution of the nonstationary pearl pulsations in terms of proton drifts depending on magnetic activity, and evaluate the magnetospheric electric field based on the variation of the central frequency of pearl pulsations.
\end{abstract}

Key words: Magnetospheric physics (magnetospheric configuration and dynamics; plasma waves and instabilities)

\section{Introduction}

Electromagnetic ion cyclotron (EMIC) waves in the form of Pc1 pulsations have been extensively studied in the past (for reviews, see e.g. Guglielmi and Pokhotelov, 1996; Kangas et al., 1998). Among the most common and intriguing types of Pcl pulsations are the pearl pulsations which consist of periodic emissions in the form of wave trains or "pearls" (Sucksdorff, 1936). They are thought to be wave packets propagating along the magnetic field line between conjugate ionospheres

Correspondence to: K. Mursula e-mail: kalevi.mursula@oulu.fi
(Jacobs and Watanabe, 1964; Obayashi, 1965). EMIC waves are a consequence of gyroresonant interaction with energetic protons (Cornwall, 1965; Kennel and Petschek, 1966; Feygin and Yakimenko, 1969; Gendrin et al., 1971). In spite of a fairly good understanding of the properties and generation mechanisms of these pulsations their relation to other geomagnetic perturbations is still insufficiently investigated. The results obtained by Wentworth (1964), Plyasova-Bakounina and Matveeva (1968), and Feygin et al. (1970) show that the majority of pearl pulsations was observed during the recovery phase of a magnetic storm. The relation of temporal variations in Pcl pulsations to geomagnetic activity was studied in detail by Fraser (1968).

The analysis by Matveeva et al. (1972) revealed that in most cases the pulsation central frequency decreases towards the end of the pearl series. Campbell (1967) showed that during the diurnal variation, the Pc1 frequency increases during very early morning hours and decreases before noon. He proposed that this phenomenon may be connected with different ionospheric attenuation of hydromagnetic waves.

This study is devoted to the analysis of long-lasting ( 2-6 h) pearl pulsations with decreasing, increasing, or constant central frequency, and to the connection of non-stationary pearl pulsations with nightside substorm activity (measured by $A E$-index). On the other hand, the stationary series of pearl pulsations appear mainly during quiet magnetic conditions. The work is organized in the following way. The observational results and the corresponding data analysis are presented in Sect. 2. We discuss the observations and present a theoretical interpretation in Sect. 3. Our conclusions are found in Sect. 4.

\section{Observations and data analysis}

We have analysed Pc1 pearls observed at three Finnish stations (Ivalo, $\lambda=110^{\circ}, \Phi=64.9^{\circ}, L=5.5$, Sodankylä, $\lambda=109^{\circ}, \Phi=63.9^{\circ}, L=5.1$, and Oulu, $\lambda=$ $107^{\circ}, \Phi=61.8^{\circ}, L=4.3$ ) between $1975-1995$. The 
local time at these observational sites is connected to universal time roughly by $L T=U T+2 \mathrm{~h}$. We divide the Pcl pearl events into two categories according to the temporal evolution of central frequency $f_{0}$ : (a) nonstationary events with increasing or decreasing $f_{0}$ (65 events), and (b) stationary events with constant $f_{0}$ (50 events).

The average duration of all events is about $2.5 \mathrm{~h}$, and the mean variation of $f_{0}$ relative to the starting frequency for nonstationary events is about $30 \%$. The average $K_{p}$ was about 2-3 for nonstationary events and 1 for stationary events. We note that variation of the central frequency is always accompanied by a change in the repetition period $\tau$. During the events with decreasing (increasing) central frequency $\tau$ increases (decreases) from the beginning to the end of the event. We may assume that the increase (decrease) in $\tau$ may be produced by the drift of the Pcl source to the region of longer (shorter) geomagnetic field lines where the external magnetic field is weaker (stronger). Moreover, at the beginning of events with decreasing $f_{0}$ the product $\tau f$ (average $\left.(\tau f)_{s t}=104\right)$ is always greater than at the end (average $(\tau f)_{\text {end }}=90$ ). The opposite variation was observed for events with increasing central frequency. Of course, a more detailed statistical treatment of the problem in hand is necessary, using quantitative parameters such as standard deviation, confidence level, error bars etc. However, this is outside the scope of the present study and a quantitative analysis will be given in a separate paper.

Typical examples of non-stationary events are shown in Figs. 1-3. These events characterise the general features which are common for events included in this analysis. Figure 1 demonstrates an event with decreasing $f_{0}$, Fig. 2 an event with increasing $f_{0}$, and Fig. 3 an event with an arc-like variation of $f_{0}$. The Pcl pearl event of Fig. 1 is characterised by the following features. The initial central frequency $f_{s t}$ was $1.36 \mathrm{~Hz}$, and the final central frequency $f_{\text {end }}$ was $0.75 \mathrm{~Hz}$. The repetition period $\tau$ varied from $\tau_{s t}=111 \mathrm{~s}$ at the beginning of event to $\tau_{\text {end }}=166 \mathrm{~s}$ at the end. The product $\tau f$ varied from $(\tau f)_{s t}=150$ to $(\tau f)_{\text {end }}=125$. In the event shown in Fig. 2 the corresponding values were $f_{s t}=1.0 \mathrm{~Hz}$, $f_{\text {end }}=1.36 \mathrm{~Hz}, \tau_{s t}=88 \mathrm{~s}, \tau_{\text {end }}=70 \mathrm{~s}, \quad(\tau f)_{s t}=88$, and $(\tau f)_{\text {end }}=95$.

As a rule, the nonstationary events were observed during moderate geomagnetic activity with $A E \leq$ $500 \mathrm{nT}$. In many cases the decrease in central frequency was accompanied by a growth in the $A E$-index. The latter means that there was a substorm activity developing within the nightside of the magnetosphere. It should be noted that, typically, Pc1 generation was terminated when the $A E$-index approached its maximum value. Figure 4 shows the $A E$-index variation on February 5, 1993, accompanied by nonstationary (04-08 UT) and stationary (12-14 UT) Pc1 pulsations. (Standard 1-h $A E$-index values provided by Kyoto WDC-3 were used in our analysis). The central frequency of the nonstationary pulsations in Fig. 4 varied from $0.8 \mathrm{~Hz}$ to $0.5 \mathrm{~Hz}$. The $A E$-index during this period increased from the value 90 to 290 , whereas during the stationary event $\left(f_{0}=0.6 \mathrm{~Hz}\right)$ it remained constant at $A E \simeq 60$.

Figure 5 shows schematically the diurnal variation of the occurrence of nonstationary Pc1s. Events with decreasing frequency are observed mostly in the late morning and around noon while events with increasing central frequency are observed either soon after midnight or in the afternoon. Because Pcl pulsations with decreasing central frequency were observed during periods of rising auroral magnetic activity, it is important to consider how this reduction in the wave frequency depends on the $A E$ index. Figure 6 represents the frequency variation of the late morning and prenoon nonstationary Pc1s with $A E$. In all cases $A E$ at the beginning was smaller than at the end of the event (leading to the negative slope in Fig. 6). Most events started under relatively low magnetic activity with $A E$ smaller than $150 \mathrm{nT}$. The rate of frequency decrease was higher for periods of low $A E$ values than for periods
SODANKYLÄ 29.10.1994

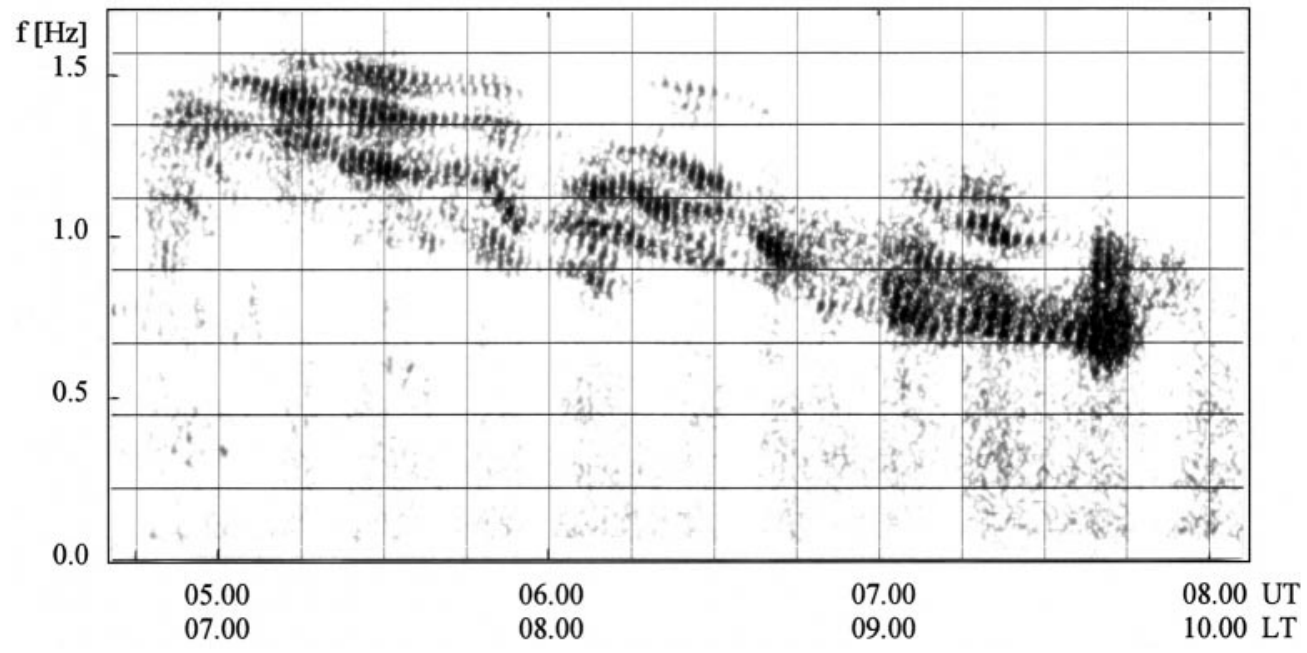

Fig. 1. The sonagram of Pcl pulsations observed at Sodankylä on October 29, 1994, showing a decreasing central frequency 
OULU 17.10.1986

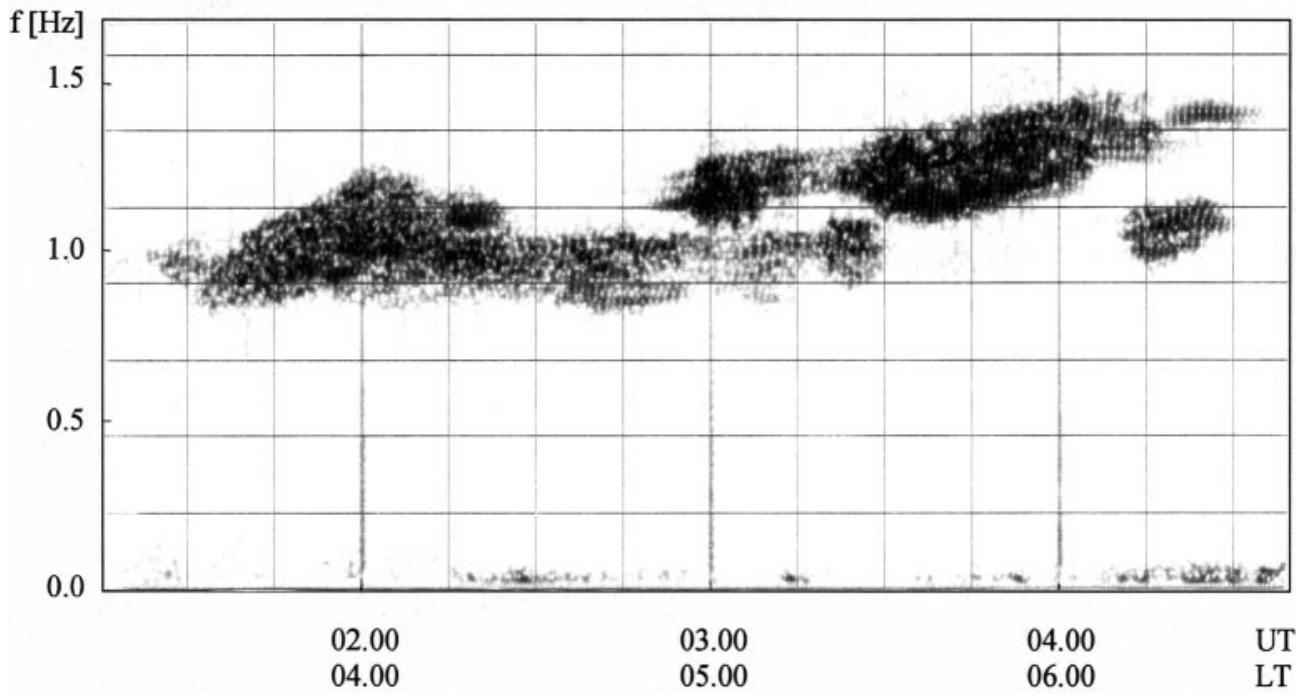

Fig. 2. The sonagram of Pcl pulsations observed at Oulu on October 17, 1986, showing an increasing central frequency
SODANKYLÄ 20.2.1995

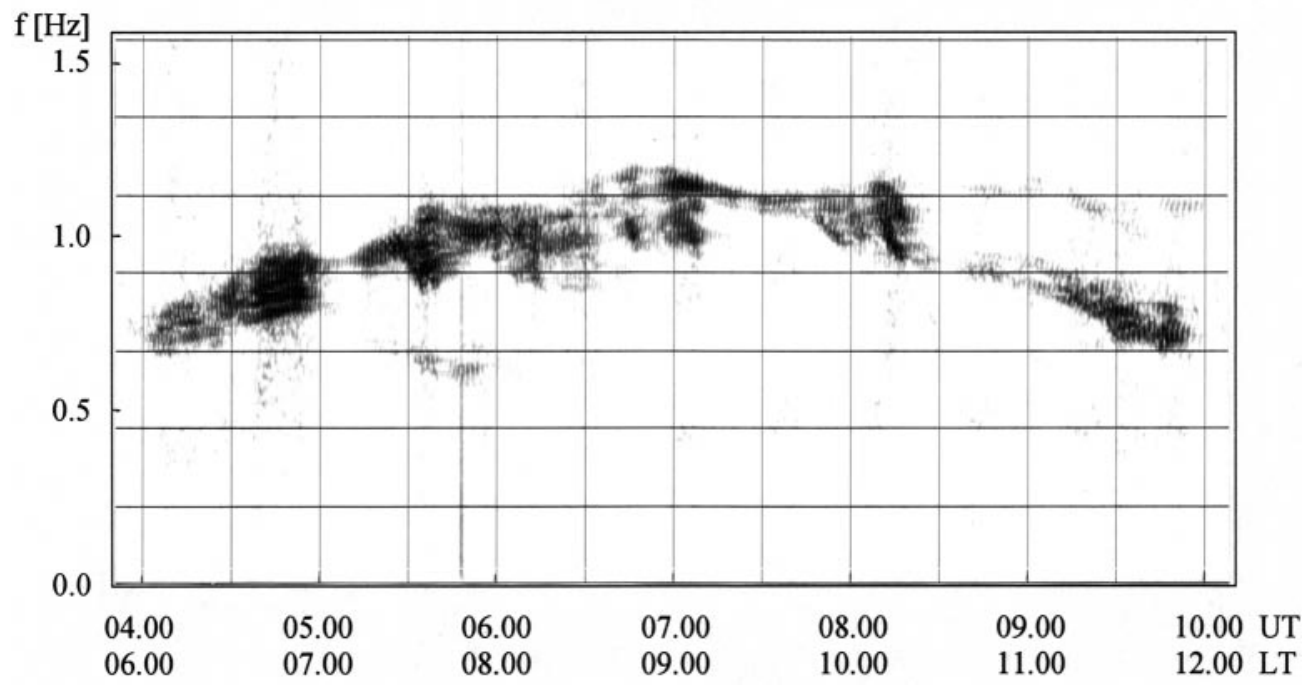

Fig. 3. The sonagram of Pc1 pulsations observed at Sodankylä on February 20, 1995, illustrating a case when both an increase and a decrease in the central frequency appears during the same event with $A E$ greater $300 \mathrm{nT}$. No dependence between the final frequencies of the individual events and $A E$ values was found. On the other hand, contrary to the morning Pc1s with decreasing frequency depicted in Fig. 6, the

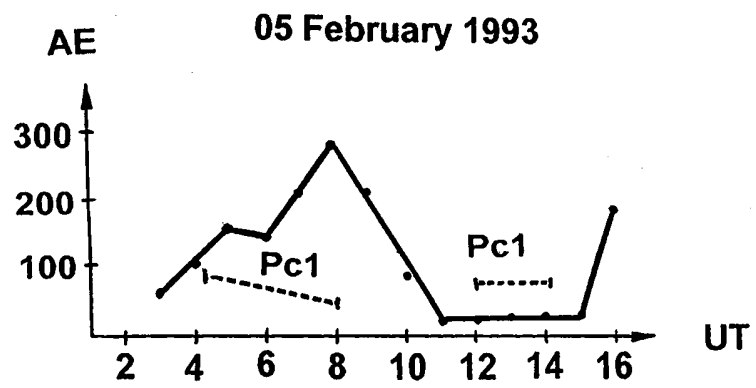

Fig. 4. Temporal variation of the $A E$-index (solid line) and the trend of the central frequency (dashed lines) of nonstationary and stationary pearl pulsations observed at Sodankylä on February 5, 1993 afternoon Pcl pulsations with increasing central frequency show no coherent pattern with $A E$, and have been observed during periods of both rising and decreasing $A E$ values.

Figure 3 illustrates a situation when the central frequency of structured Pcl pulsations first increased and than decreased. It is worth mentioning that this event coincides with a similar temporal variation of the $H$-component of the geomagnetic field (as measured, e.g. by the normal magnetogram at Sodankylä; not shown). The geomagnetic conditions were very quiet during this event $\left(K_{p}=1-2\right)$. The central frequency increased from $0.75 \mathrm{~Hz}$ to $1 \mathrm{~Hz}$ between $04.00 \mathrm{UT}$ and $07.00 \mathrm{UT}$ and then decreased from $1 \mathrm{~Hz}$ to $0.75 \mathrm{~Hz}$ between $07.00 \mathrm{UT}$ and 10.00 UT. At the beginning of the event $(\sim 04 \mathrm{UT}) \tau_{s t}=97 \mathrm{~s}$, in the middle $(\sim 07 \mathrm{UT})$ $\tau_{\text {middle }}=83 \mathrm{~s}$, and at the end of the series $(\sim 10 \mathrm{UT})$ $\tau_{\text {end }}=104 \mathrm{~s}$. Correspondingly, $(\tau f)_{\text {st }}=78,(\tau f)_{\text {middle }}$ $=83$ and $(\tau f)_{\text {end }}=78$. 


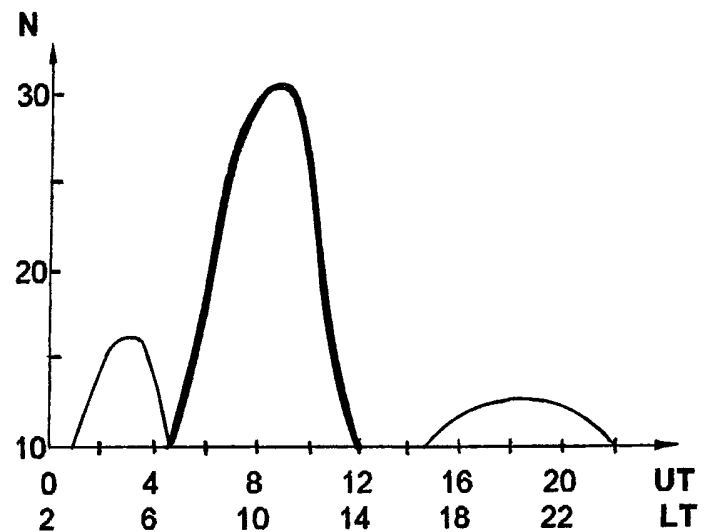

Fig. 5. The diurnal variation of the occurrence of Pc1s with decreasing central frequency (bold line) and with increasing frequency (thin line)

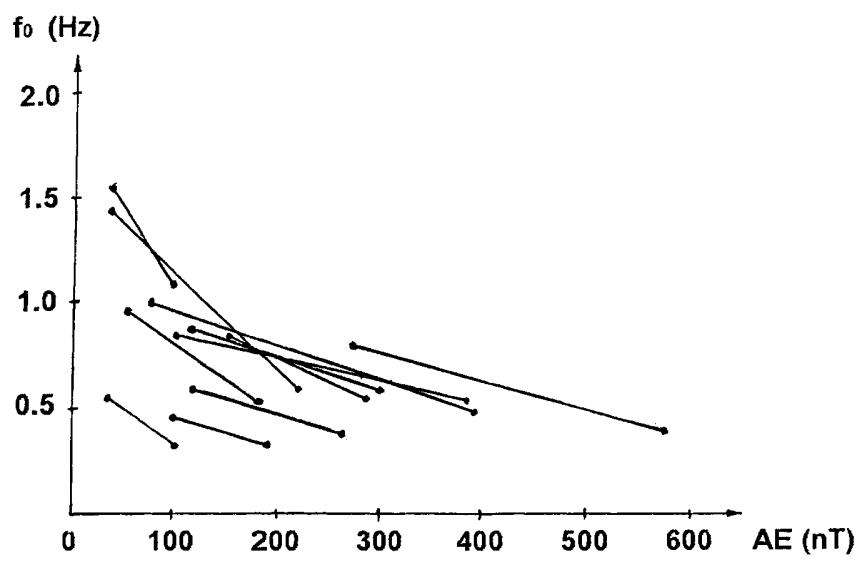

Fig. 6. The frequency variation of the late morning and prenoon nonstationary pulsations with $A E$-index

Now let us consider events with time-independent $f_{0}$. We have investigated about 50 cases which are characterised by a constant central frequency and a constant repetition period during the event. All such events were observed during very low magnetic activity $\left(K_{p} \approx 1\right)$. A typical example of such a Pcl pearl event is shown in Fig. 7. This event is characterised by $f_{0}=0.54 \mathrm{~Hz}$, $\tau=290 \mathrm{~s}$ and $K_{p}=1$.

\section{Discussion}

The diurnal variation of the nonstationary Pcl events shows that the events exhibiting a decrease in the central frequency are mainly observed in the late morning and at noon, while those with increasing central frequency appear after midnight and in the afternoon. The idea which allows us to understand the diurnal distribution of nonstationary Pcls is connected with the drift of energetic protons in the equatorial plane during substorm activity. The trajectories of protons drifting in the magnetosphere depend on convection and corotation electric fields as well as on the proton diamagnetic drift. The convection electric field $\mathbf{E}$ in the dawn-dusk direction is increased near the 23.00 LT with a longitu- dinal extent of several hours (Nishida, 1978). During the substorm growth phase this field gradually increases. The protons injected in the night sector undergo the $\mathbf{E} \times \mathbf{B}$ drift to the region of increasing magnetic field (towards the Earth). This longitudinal sector extends from early morning to late evening hours. In the dayside the magnetospheric dawn-dusk electric field produces the drift of energetic protons to the region of decreasing magnetic field (outward drift). The calculations of entire trajectories of protons moving due to the $\mathbf{E} \times \mathbf{B}$ and diamagnetic drifts after injection in the nightside have been provided by Chen (1970), and Lyons and Samson (1992). These authors showed that during the early morning and late evening hours the protons are drifting towards the Earth, i.e. to the region of increased magnetic field, while in the day sector they move predominantly outwards (from the Earth).

Let us now consider parameters which control the variation of the Pcl central frequency. The latter corresponds to the maximum of the wave growth rate (Cornwall, 1965) given by

$f_{0} \approx f_{p} c_{A} / v_{T}$,

where $f_{p}$ is the equatorial proton gyrofrequency, $c_{A}$ the Alfvén velocity and $v_{T}$ the proton thermal velocity.

According to Eq. (1) the central frequency depends on the $L$-shell value (the McIllwain parameter). To demonstrate this we note that $f_{p} \propto B$, and $c_{A} \propto B / \sqrt{n}$, where $n$ is the plasma number density. For adiabatic motions the thermal proton velocity scales as $\sqrt{B}$. On the other hand, the plasma number density varies in the dayside magnetosphere as $n \propto L^{-2}$ for a saturated plasmasphere and $n \propto L^{-4.5}$ for plasma trough (Carpenter and Anderson, 1992). Assuming a dipole field geometry, the magnetic field intensity scales as $\propto L^{-3}$. Using these scaling laws we obtain the following approximate variation for the central frequency with $L: f_{0} \propto L^{-3.5}$ for saturated plasmasphere and $f_{0} \propto L^{-2.25}$ for plasma trough.

The dependence of the central frequency on $L$-value allows us to evaluate the value of the electric field using the observed variation of pearl pulsation frequency (Troitskaya et al., 1968; Lacourly, 1969). We assume that the variation of the central frequency arises from the radial drift of $\mathrm{Pcl}$ source across $L$-shells with velocity $\left|v_{d}\right|=E / B$. The latter can be estimated as $\left|v_{d}\right|=R_{E} d_{t} L$, where $R_{E}$ is the Earth's radius and $d_{t}$ stands for the derivative $d / d t$. Taking the time derivative from Eq. (1) we obtain

$\left|v_{d}\right|=\left(R_{E} L / 3.5\right) d_{t} \ln f_{0}$,

for the saturated plasmasphere and

$\left|v_{d}\right|=\left(R_{E} L / 2.25\right) d_{t} \ln f_{0}$,

for the plasma trough profile.

With the help of Eqs. (2) and (3) the electric field (in $\mathrm{V} / \mathrm{m})$ can now be evaluated as

$E \approx\left(\alpha / L^{2}\right) d_{t} \ln f_{0}$,

where $\alpha \simeq 60$ for the saturated plasmasphere and $\alpha \simeq 90$ for the plasma trough. 
IVALO 21.12.1995

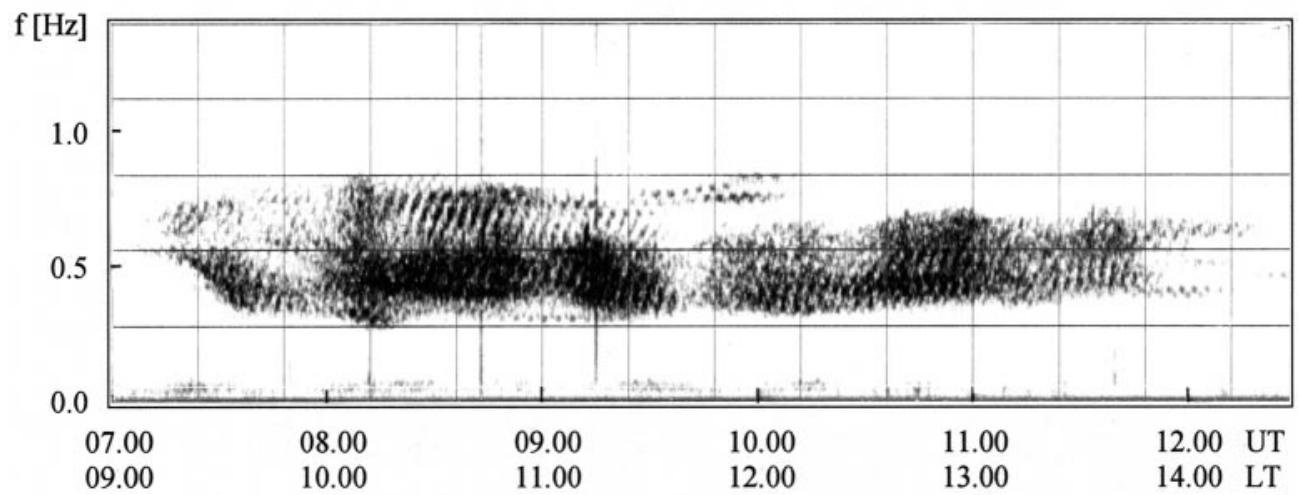

Fig. 7. The sonagram of $\mathrm{Pcl}$ pulsations observed at Ivalo on December 21, 1995, depicting a quasi-stationarycentralfrequency and repetition structure
From Figs. 1 and 2 for $L=5$ we obtain $E \approx 10^{-4} \mathrm{~V} / \mathrm{m}$ for saturated plasmasphere and $E \approx 1.5 \times 10^{-4} \mathrm{~V} / \mathrm{m}$ for the plasma trough. The values for $E$ in the equatorial plane of the magnetosphere correspond to the average electric fields measured by GEOS-1 (Pedersen et al., 1978). These authors noted that local regions exist where electric fields and corresponding plasma flows have values above the average. Such phenomena may normally be responsible for the formation of nonstationary Pc1 pulsations during substorm activity.

\section{Conclusions}

We have discussed the formation of nonstationary Pc1 pulsations and shown that nonstationary Pcls are related to simultaneous magnetic activity. The events exhibiting a decrease in wave frequency are mostly observed in the late morning and at noon, while events with increasing frequency are observed either in the post-midnight to early morning hours, or in late afternoon hours. Such a daily variation is probably due to the impact of quasi-static electric fields on the motion of the Pcl source. In fact, the existence of the dawn-to-dusk electric field leads to the motion of the Pcl source away from the Earth in the dayside magnetosphere and towards to the Earth in the nightside. This results in a decrease in Pcl central frequency in the dayside and to a corresponding increase during early morning and late evening hours. The behaviour of $\tau$ during the events also agrees with this scenario. We evaluated the magnetospheric electric field $E$ responsible for the movement of the Pc1 source and found the value of $E \approx(0.5 \div 2.5) \times 10^{-4} \mathrm{~V} / \mathrm{m}$ which is in reasonable agreement with the average electric fields measured by the GEOS-1 satellite. Thus, we have suggested and derived an interesting result (magnetospheric diagnosis) that it is possible to extract an indirect estimate of the convection electric field in the equatorial magnetosphere using ground observations of Pcl pearl pulsations.

Acknowledgements. We acknowledge financial support from the Academy of Finland, and the Commission of the European Union (grant no. INTAS-96-2064). One of us (O.A.P.) wishes to thank the French Ministère de la Recherche et de la Technologie for hospitality at LPCE where this work was completed. The authors wish to thank Prof. A. V. Guglielmi and Dr. A. S. Potapov for valuable comments.

Topical Editor G. Chanteur thanks M. Balikhin, K. Hayashi and S. Perraut for their help in evaluating this paper.

\section{References}

Campbell, W. H., Low attenuation of hydromagnetic waves in the ionosphere and implied characteristics in the magnetosphere for Pc1 events, J. Geophys. Res., 72, 3429-3446, 1967.

Carpenter, D. L., and R. R. Anderson, An ISEE/whistler model of equatorial electron density in the magnetosphere, J. Geophys. Res., 97, 1097-1108, 1992.

Chen, A. J., Penetration of low-energy protons deep into the magnetosphere, J. Geophys. Res., 75, 2458-2467, 1970.

Cornwall, J. M., Cyclotron instability and electromagnetic emission in the ultra low frequency and very low frequency ranges, J. Geophys. Res., 70, 61-69, 1965.

Feygin, F. Z., and V. L. Yakimenko, Mechanism of "pearl" generation and development by cyclotron instability of the outer proton zone, Geomagn. Aeron., 9, 565-569, 1969.

Feygin, F. Z., M. B. Gokhberg, and E. T. Matveeva, Comparison of satellite data with the occurrence of Pc1 pulsations, Ann. Geophysicae, 26, 903-906, 1970.

Fraser, B. J., Temporal variations in Pcl geomagnetic pulsations, Planet. Space Sci., 16, 111-124, 1968.

Gendrin, R., S. Lacourly, A. Roux, J. Solomon, F. Z. Feygin, M. B. Gokhberg, V. A. Troitskaya, and V. L. Yakimenko, Wave packet propagation in the amplifying medium and its application to the dispersion characteristics and to the generation mechanisms of Pc1 events, Planet. Space Sci., 19, 165-194, 1971.

Guglielmi, A. V., and O. A. Pokhotelov, Geoelectromagnetic waves, IOP Publishers, Bristol, 1996.

Jacobs, J. A., and T. Watanabe, Micropulsation whistlers, J. Atmos. Terr. Phys., 26, 825-829, 1964.

Kangas, J., A. V. Guglielmi, and O. A. Pokhotelov, Morphology and physics of short-period magnetic pulsations (a review), Space Sci. Rev., 83, 435-512, 1998.

Kennel, C. F., and H. E. Petschek, Limit on stably trapped particles fluxes, J. Geophys. Res., 71, 1-28, 1966.

Lacourly, S., Evaluation de certain parametrès de la magnètosphere a partir des propriètès des pulsations hydromagnètiques irrègulières (S.I.P. et I.P.D.P.), Ann. Geophysicae, 25, 651-657, 1969.

Lyons, L. R., and J. C. Samson, Formation of the stable auroral arc that intensifies at substorm onset, Geophys. Res. Lett., 19, 21712174, 1992.

Matveeva, E. T., A. L. Kalisher, and B. V. Dovbnya, Physical conditions in the magnetosphere and the interplanetary space during excitation of type Pcl geomagnetic pulsations, Geomagn. Aeron., 12, 977-978, 1972. 
Nishida, A., Geomagnetic diagnosis of the magnetosphere, Springer, New York Heidelberg Berlin, 1978.

Obayashi, T., Hydromagnetic whistlers, J. Geophys. Res., 70, 1069-1078, 1965.

Pedersen, A., R. Grard, K. Knott, D. Jones, and A. Gonfalone, Measurements of quasi-static electric fields between 3 and 7 Earth radii on GEOS-1, Space Sci. Rev., 22, 333-346, 1978.

Plyasova-Bakounina, T. A., and E. T. Matveeva, Relationship between pulsations of Pc 1 type and magnetic storms, Geomagn. Aeron., 8, 153-155, 1968.
Sucksdorff, E., Occurrences of rapid micropulsations at Sodankylä during 1932 to 1935, Terrestr. Magn. Atmosp. Electr., 41, 337344, 1936.

Troitskaya, V. A., R. V. Schepetnov, and A. V. Guglielmi, Estimate of electric fields in the magnetosphere from the frequency drift of micropulsations, Geomagn. Aeron., 8, 634-635, 1968.

Wentworth, R. C., Enhancement of hydromagnetic emissions after geomagnetic storms, J. Geophys. Res., 69, 2291-2298, 1964. 